PROCEEDINGS OF THE

AMERICAN MATHEMATICAL SOCIETY

Volume 125, Number 4, April 1997, Pages 1209-1214

S 0002-9939(97)03622-8

\title{
INTERSECTION OF SETS WITH $n$-CONNECTED UNIONS
}

\author{
CHARLES D. HORVATH AND MARC LASSONDE
}

(Communicated by Peter Li)

\begin{abstract}
We show that if $n$ sets in a topological space are given so that all the sets are closed or all are open, and for each $k \leq n$ every $k$ of the sets have a $(k-2)$-connected union, then the $n$ sets have a point in common. As a consequence, we obtain the following starshaped version of Helly's theorem: If every $n+1$ or fewer members of a finite family of closed sets in $\mathbb{R}^{n}$ have a starshaped union, then all the members of the family have a point in common. The proof relies on a topological KKM-type intersection theorem.
\end{abstract}

\section{INTRODUCTION}

The purpose of this paper is to generalize to an arbitrary finite family of sets the following elementary fact: If in a topological space two nonempty sets, both closed or both open, have a pathwise connected union, then they have a point in common. To this end, we use the notion of $n$-connectedness which is a natural generalization of pathwise connectedness. Let us recall the definition.

For any integer $n \geq-1$, we denote by $\Delta_{n+1}$ the unit $(n+1)$-simplex and by $\partial \Delta_{n+1}$ its boundary. A topological space $C$ is said to be $n$-connected if every continuous map $f: \partial \Delta_{n+1} \rightarrow C$ has a continuous extension $g: \Delta_{n+1} \rightarrow C$.

Clearly, $(-1)$-connected means nonempty and 0 -connected means pathwise connected. It is also easily seen that for a pathwise connected space, 1-connected is equivalent to simply connected. Convex sets and starshaped sets in topological vector spaces, and, more generally, contractible spaces are $n$-connected for every $n$.

Our main result (Theorem 3) reads as follows: If $n$ sets in a topological space are given so that all the sets are closed or all are open and for each $k \leq n$ every $k$ of the sets have $a(k-2)$-connected union, then the $n$ sets have a point in common. For $n=2$ we exactly recover the above-mentionned fact. This extension is equivalent to Brouwer's fixed point theorem.

Applications to Helly-type theorems are considered. In particular, the following generalization of results of Breen [2,3] is established (Theorem 5): If every $n+1$ or fewer members of a finite family of closed sets in $\mathbb{R}^{n}$ have a starshaped union, then all the members of the family have a point in common.

The basic tool for our study is an intersection theorem (Theorem 2) obtained by combining the well-known theorem of Knaster-Kuratowski-Mazurkiewicz with a selection-extension property for families of $n$-connected sets (Theorem 1). No

Received by the editors August 14, 1995 and, in revised form, October 25, 1995.

1991 Mathematics Subject Classification. Primary 52A30, 54C99; Secondary 52A35, 52A07.

Key words and phrases. $n$-connected sets, starshaped sets, Helly's theorem, KKM theorem.

(C)1997 American Mathematical Society 
notion from algebraic topology is used. The ideas of this approach come from Horvath [11] and Lassonde [14].

For a comprehensive account on Helly-type results, the reader is referred to the survey papers of Danzer, Grünbaum, Klee [4] and Eckhoff [7].

\section{SELECTION-EXTENSION PROPERTY}

By polytope we understand a simplicial CW-complex in the sense of Whitehead. We recall the definitions. A triangulation is a collection $\mathcal{T}$ of geometric closed simplexes such that every face of a simplex in $\mathcal{T}$ is itself a simplex in $\mathcal{T}$, and the intersection of any two simplexes in $\mathcal{T}$ is a face of both of them. We denote by $\mathcal{T}^{(n)}$ the collection of all simplexes of $\mathcal{T}$ of dimension less than or equal to $n$.

A polytope is a topological space $P$ together with a triangulation $\mathcal{T}$ such that $P=\bigcup\{\sigma \mid \sigma \in \mathcal{T}\}$, and $P$ is supplied with the CW-topology, i.e. $U \subseteq P$ is open if and only if for each $\sigma \in \mathcal{T}, U \cap \sigma$ is open in the Euclidean topology of $\sigma$. We denote by $P^{(n)}$ the $n$-skeleton of $P$, i.e. the subpolytope of $P$ with triangulation $\mathcal{T}^{(n)}$. A subpolytope of $P$ is a polytope $Q \subseteq P$ with a triangulation $\mathcal{S} \subseteq \mathcal{T}$.

The following theorem, though more general than required for the applications considered in this paper, is worth stating explicitly for future use. It strengthens a result of Horvath [11, Theorem 1] in several aspects; the proof is similar.

Theorem 1. Let $X$ be a topological space, $P$ a polytope with triangulation $\mathcal{T}, Q \subseteq$ $P$ a subpolytope with triangulation $\mathcal{S} \subseteq \mathcal{T}$, and $\left\{C_{\sigma} \mid \sigma \in \mathcal{T}\right\}$ a family of subsets of $X$ such that:

(a) $C_{\sigma} \subseteq C_{\tau}$ for every $\sigma, \tau \in \mathcal{T}$ with $\sigma \subseteq \tau$,

(b) $C_{\sigma}$ is $($ dim $\sigma-1)$-connected for every $\sigma \in \mathcal{T} \backslash \mathcal{S}$.

Then any continuous map $f: Q \rightarrow X$ satisfying $f(\sigma) \subseteq C_{\sigma}$ for all $\sigma \in \mathcal{S}$ can be extended to a continuous map $g: P \rightarrow X$ satisfying $g(\sigma) \subseteq C_{\sigma}$ for all $\sigma \in \mathcal{T}$.

Proof. Since for any vertex $x \in \mathcal{T}^{(0)}$ the set $C_{\{x\}}$ is not empty because of (b), the map $f_{0}: Q \cup P^{(0)} \rightarrow X$ given by $f_{0}(x)=f(x)$ for $x \in Q$, and $f_{0}(x)=$ any point in $C_{\{x\}}$ for $x \in P^{(0)} \backslash Q$ is a continuous extension of $f$ such that $f_{0}(\sigma) \subseteq C_{\sigma}$ for all $\sigma \in \mathcal{S} \cup \mathcal{T}^{(0)}$. Starting from $f_{0}$, we define inductively a sequence of continuous extensions $f_{n}: Q \cup P^{(n)} \rightarrow X$ satisfying $f_{n}(\sigma) \subseteq C_{\sigma}$ for all $\sigma \in \mathcal{S} \cup \mathcal{T}^{(n)}$. The result then follows by taking $g: P \rightarrow X$ such that $g(x)=f_{n}(x)$ whenever $x \in Q \cup P^{(n)}$.

Suppose $f_{n}$ is constructed. The required extension of $f_{n}$ is obtained by pasting together a family of maps $f_{n+1}^{\sigma}: \sigma \rightarrow C_{\sigma}, \sigma \in \mathcal{S} \cup \mathcal{T}^{(n+1)}$, defined as follows. For $\sigma \in \mathcal{S} \cup \mathcal{T}^{(n)}$, simply let $f_{n+1}^{\sigma}=f_{n \mid \sigma}$. Otherwise, write $\partial \sigma=\sigma_{1} \cup \ldots \cup \sigma_{k}$, where each $\sigma_{i}$ belongs to $\mathcal{T}^{(n)}$; for each $i=1, \ldots, k$, we have $f_{n}\left(\sigma_{i}\right) \subseteq C_{\sigma_{i}}$ by construction and $C_{\sigma_{i}} \subseteq C_{\sigma}$ by (a), so that $f_{n}(\partial \sigma) \subseteq C_{\sigma}$ : let $f_{n+1}^{\sigma}: \sigma \rightarrow C_{\sigma}$ be a continuous extension of $f_{n \mid \partial \sigma}: \partial \sigma \rightarrow C_{\sigma}$ (which exists because of (b)). Since for any different members $\sigma, \tau$ in $\mathcal{S} \cup \mathcal{T}^{(n+1)}$ we have $f_{n+1 \mid \sigma \cap \tau}^{\sigma}=f_{n \mid \sigma \cap \tau}=f_{n+1 \mid \sigma \cap \tau}^{\tau}$, there is a map $f_{n+1}: Q \cup P^{(n+1)} \rightarrow X$ which is an extension of each $f_{n+1}^{\sigma}$. Obviously, such a map is continuous for the CW-topology, extends $f_{n}$, and satisfies $f_{n+1}(\sigma) \subseteq C_{\sigma}$ for all $\sigma \in \mathcal{S} \cup \mathcal{T}^{(n+1)}$.

As an immediate special case of Theorem 1, we mention the following well-known property (see for example Eilenberg [8, p. 241]): 
Corollary. A topological space $X$ is $k$-connected for every $k \leq n$ if and only if for any polytope $P$ and any subpolytope $Q \subseteq P$, any continuous $f: Q \rightarrow X$ extends continuously over $Q \cup P^{(n+1)}$.

Proof. The "if part" is obvious, and the "only if part" follows from Theorem 1 applied to the polytope $Q \cup P^{(n+1)}$ and the sets $C_{\sigma}$ given by $C_{\sigma}=X$ for every $\sigma \in \mathcal{S} \cup \mathcal{T}^{(n+1)}$.

\section{KKM-TYPE INTERSECTION THEOREMS}

We denote by $\mathcal{F}\left(\Delta_{n}\right)$ the set of all faces of $\Delta_{n}$, and for any $\sigma \in \mathcal{F}\left(\Delta_{n}\right)$ we denote by $\sigma^{(0)}$ the set of all vertices of $\sigma$. The Knaster-Kuratowski-Mazurkiewicz theorem can be formulated as follows: If a family $\left\{A_{i} \mid i \in \Delta_{n}^{(0)}\right\}$ of subsets of $\Delta_{n}$ is such that all the sets are closed or all are open, and each face $\sigma$ of $\Delta_{n}$ is contained in the corresponding union $\bigcup\left\{A_{i} \mid i \in \sigma^{(0)}\right\}$, then there is a point common to all the sets. (The original statement deals only with closed sets; a proof that open sets may be used equivalently is given in Lassonde [14].)

By combining Theorem 1 with the Knaster-Kuratowski-Mazurkiewicz theorem we get our basic topological intersection theorem:

Theorem 2. In a topological space, let $\left\{A_{i} \mid i \in \Delta_{n}^{(0)}\right\}$ be a family of sets, all closed or all open, and let $\left\{C_{\sigma} \mid \sigma \in \mathcal{F}\left(\Delta_{n}\right)\right\}$ be an associated family of sets such that:

(a) $C_{\sigma} \subseteq C_{\tau}$ for every $\sigma, \tau \in \mathcal{F}\left(\Delta_{n}\right)$ with $\sigma \subseteq \tau$,

(b) $C_{\sigma}$ is $(\operatorname{dim} \sigma-1)$-connected for every $\sigma \in \mathcal{F}\left(\Delta_{n}\right)$.

Then the following equivalent assertions hold:

(1) If each $C_{\sigma}$ is contained in the corresponding union $\bigcup\left\{A_{i} \mid i \in \sigma^{(0)}\right\}$, then $C_{\Delta_{n}}$ contains a point of the intersection $\bigcap\left\{A_{i} \mid i \in \Delta_{n}^{(0)}\right\}$;

(2) If $C_{\Delta_{n}}$ is contained in the union $\bigcup\left\{A_{i} \mid i \in \Delta_{n}^{(0)}\right\}$, then some $C_{\sigma}$ contains a point of the corresponding intersection $\bigcap\left\{A_{i} \mid i \in \sigma^{(0)}\right\}$.

Proof. Denote by $X$ the underlying topological space, and assume that the condition of assertion (1) is satisfied. By Theorem 1, a continuous $g: \Delta_{n} \rightarrow X$ exists such that for each face $\sigma$ of $\Delta_{n}$ we have $g(\sigma) \subseteq C_{\sigma}$, which in view of the assumption implies $g(\sigma) \subseteq \bigcup\left\{A_{i} \cap C_{\Delta_{n}} \mid i \in \sigma^{(0)}\right\}$. Thus, each face $\sigma$ of $\Delta_{n}$ is contained in the corresponding union $\bigcup\left\{g^{-1}\left(A_{i} \cap C_{\Delta_{n}}\right) \mid i \in \sigma^{(0)}\right\}$. We derive from the theorem of Knaster-Kuratowski-Mazurkiewicz that the family $\left\{g^{-1}\left(A_{i} \cap C_{\Delta_{n}}\right) \mid i \in \Delta_{n}^{(0)}\right\}$ has a nonempty intersection. Consequently, the intersection $\bigcap\left\{A_{i} \cap C_{\Delta_{n}} \mid i \in \Delta_{n}^{(0)}\right\}$ is not empty, proving assertion (1).

As for assertion (2), it suffices to observe that it is the contraposition form of assertion (1) applied to the family $\left\{X \backslash A_{i} \mid i \in \Delta_{n}^{(0)}\right\}$.

Ky Fan's extension of Knaster-Kuratowski-Mazurkiewicz's theorem [9] and Ky Fan's matching theorem [10] are obtained respectively from assertion (1) and assertion (2) of the following:

Corollary. In a convex subset of a vector space supplied with the finite topology, let $\left\{A_{i} \mid i \in I\right\}$ be a finite family of sets, all closed or all open, and let $\left\{x_{i} \mid i \in I\right\}$ be a family of points, indexed by the same set $I$. Then the following (equivalent) assertions hold: 
(1) If for every nonempty subset $J \subseteq I$ the convex hull of $\left\{x_{j} \mid j \in J\right\}$ is contained in the corresponding union $\bigcup\left\{A_{j} \mid j \in J\right\}$, then the convex hull of $\left\{x_{i} \mid i \in I\right\}$ contains a point of the intersection $\bigcap\left\{A_{i} \mid i \in I\right\}$.

(2) If the convex hull of $\left\{x_{i} \mid i \in I\right\}$ is contained in the union $\bigcup\left\{A_{i} \mid i \in I\right\}$, then for some nonempty subset $J \subseteq I$ the convex hull of $\left\{x_{j} \mid j \in J\right\}$ contains a point of the corresponding intersection $\bigcap\left\{A_{j} \mid j \in J\right\}$.

Proof. We may assume that the set of indices $I$ is the set of vertices of $\Delta_{n}$. The result then follows from Theorem 2 with $C_{\sigma}$ being, for every $\sigma \in \mathcal{F}\left(\Delta_{n}\right)$, the convex hull of $\left\{x_{j} \mid j \in \sigma^{(0)}\right\}$ (such sets are evidently contractible when supplied with the Euclidean topology).

\section{KleE-TyPe intersection theorems}

Our main result below provides topological generalizations of the familiar theorem of Klee [12]: If $n$ closed convex sets in a topological vector space are such that their union is convex and the intersection of every $n-1$ of them is nonempty, then all the sets have a point in common.

Theorem 3. Let $n$ sets in a topological space be given so that all the sets are closed or all are open, and either of the following properties is satisfied:

(1) For each $k \leq n$ the union of every $k$ of the sets is $(k-2)$-connected; or

(2) The union of the $n$ sets is $(n-2)$-connected and for each $k \leq n-1$ the intersection of every $k$ of the sets is $(n-k-2)$-connected.

Then all the sets have a point in common.

Proof. Let $\left\{A_{i} \mid i \in \Delta_{n-1}^{(0)}\right\}$ be a family of $n$ sets satisfying the conditions of the theorem. We define an associated family of sets $\left\{C_{\sigma} \mid \sigma \in \mathcal{F}\left(\Delta_{n-1}\right)\right\}$ satisfying the requirements of Theorem 2 as follows. In case (1), we let $C_{\sigma}=\bigcup\left\{A_{i} \mid i \in \sigma^{(0)}\right\}$ so that assertion (1) of Theorem 2 applies. In case (2), we let $C_{\sigma}=\bigcup\left\{A_{i} \mid i \in \sigma^{(0)}\right\}$ for $\sigma=\Delta_{n-1}$ and $C_{\sigma}=\bigcap\left\{A_{i} \mid i \notin \sigma^{(0)}\right\}$ for $\sigma \neq \Delta_{n-1}$, so that assertion (2) of Theorem 2 gives the result.

The following immediate consequence of Theorem 3 is worth mentioning:

Corollary. A family of $n$ closed convex sets in a topological vector space has a nonempty intersection if (and only if) the union of the $n$ sets is $(n-2)$-connected and the intersection of every $n-1$ of them is nonempty.

This result, which is a slight modification of Klee's theorem, implies the theorem of Brouwer stating that the $n$-sphere $S^{n}$ is not n-connected. Indeed, consider the $n$-dimensional faces of $\Delta_{n+1}$ : they form a family of $n+2$ closed convex sets in $\mathbb{R}^{n+2}$, every intersection of $n+1$ of them is nonempty, but the whole intersection is empty. Hence their union, which is $\partial \Delta_{n+1}$, is not $n$-connected, so $S^{n}$ is not $n$-connected either. Since this statement is equivalent to Brouwer's fixed point theorem, which is itself equivalent to the theorem of Knaster-Kuratowski-Mazurkiewicz, we infer that the above corollary is equivalent to all these results.

Remark. Case (2) of Theorem 3 is closely related to results of Dugundji for metric spaces (see [5, Theorem 4.1] for the open version, and [6, Theorem 5.2] for the closed version). Dugundji's assumptions are: The singular $(n-2)$-homology group of the union of the $n$ sets is trivial or a torsion group, and for each $k \leq n-1$ the intersection of every $k$ of the sets is $p$-connected for every $p \leq n-k-2$. In case (2) 
of Theorem 3 the hypothesis on the union is stronger but the hypotheses on the intersections are weaker.

\section{HeLly-type intersection theOREMS}

We now turn to discussing Helly's theorem which asserts that a finite family of convex sets in $\mathbb{R}^{n}$ has a nonempty intersection if (and only if) the intersection of every $n+1$ members of the family is nonempty. As is well known, Helly's theorem can easily be proved by combining Klee's theorem with Carathéodory's theorem (see for example Berge [1, p. 173]).

Recall that a subset $C$ of a vector space is said to be starshaped if there is a point $c \in C$ such that for every $x \in C$ the segment $[c, x]$ lies in $C$. Clearly, if the intersection of convex sets is nonempty, then their union is starshaped. Hence, the following result of Breen [2] is a generalization of Helly's theorem for closed sets: $A$ finite family of closed convex sets in $\mathbb{R}^{n}$ has a nonempty intersection if (and only if) the union of every $n+1$ or fewer members of the family is starshaped. The two theorems of this section extend Breen's result in two different directions.

In our first theorem, starshapedness of the union is weakened to $n$-connectedness, and open sets are considered as well as closed sets. Like the above Klee-type results, this extension of Helly's theorem is equivalent to Brouwer's fixed point theorem.

Theorem 4. Let a finite family of convex sets in $\mathbb{R}^{n}$ be given so that all the sets are closed or all are open, and for each $k \leq n+1$ the union of every $k$ members of the family is $(k-2)$-connected. Then all the members of the family have a point in common.

Proof. It follows from Theorem 3, case (1), that the intersection of every $n+1$ members of the family is nonempty, whence the conclusion by Helly's theorem.

In our second theorem, convexity of the sets is weakened to starshapedness. The proof combines Theorem 3 with a Krasnosel'skiǐ-type theorem of Kołodziejczyk [13] stating that if every $n+1$ members of a finite family of closed sets in $\mathbb{R}^{n}$ have a starshaped union, then the union of all the members of the family is starshaped. Theorem 5 extends another result of Breen [3], which is the special case $n=2$.

Theorem 5. If every $n+1$ or fewer members of a finite family of closed sets in $\mathbb{R}^{n}$ have a starshaped union, then all the members of the family have a point in common.

Proof. By the above-mentionned theorem of Kołodziejczyk, every union of members of the family is starshaped, so the conclusion follows from Theorem 3, case (1).

\section{REFERENCES}

1. C. Berge, Espaces topologiques, fonctions multivoques, Dunod, Paris, 1966. MR 21:4401

2. M. Breen, Starshaped unions and nonempty intersections of convex sets in $\mathbb{R}^{d}$, Proc. Amer. Math. Soc. 108 (1990), no. 3, 817-820. MR 90e:52007

3. M. Breen, An intersection property for starshaped sets in the plane, Geom. Dedicata $\mathbf{3 7}$ (1991), no. 3, 317-326. MR 92e:52008

4. L. Danzer, B. Grünbaum, and V. Klee, Helly's theorem and its relatives, Convexity, Sympos. Pure Math., Vol. 7, Amer. Math. Soc., Providence, RI, 1962, pp. 101-180. MR 28:524

5. J. Dugundji, A duality property of nerves, Fund. Math. 59 (1966), 213-219. MR 34:5071

6. J. Dugundji, Maps into nerves of closed coverings, Ann. Scuola Norm. Sup. Pisa. Sci. Fis. Mat. 21 (1967), 121-136. MR 36:7112 
7. J. Eckhoff, Helly, Radon, and Carathéodory type theorems, Handbook of convex geometry (P. M. Gruber and J. M. Wills, eds.), Elsevier Science Publishers, 1993, pp. 389-447. MR 94k:52010

8. S. Eilenberg, Cohomology and continuous mappings, Ann. Math. 41 (1940), 231-251. MR $1: 222 \mathrm{~b}$

9. K. Fan, A generalization of Tychonoff's fixed point theorem, Math. Ann. 142 (1961), 305-310. MR 24:A1120

10. K. Fan, Some properties of convex sets related to fixed point theorems, Math. Ann. 266 (1984), 519-537. MR 85i:47060

11. C. Horvath, Contractibility and generalized convexity, J. Math. Anal. Appl. 156 (1991), no. 2, 341-357. MR 92f:52002

12. V. L. Klee, On certain intersection properties of convex sets, Canad. J. Math. 3 (1951), 272-275. MR 13:153m

13. K. Kołodziejczyk, On starshapedness of the union of closed sets in $\mathbb{R}^{n}$, Colloq. Math. $\mathbf{5 3}$ (1987), 193-197. MR 89b:52017

14. M. Lassonde, Sur le principe KKM, C.R. Acad. Sci. Paris Sér. I Math. 310 (1990), 573-576. MR 91h:47061

Département de Mathématiques, Université de Perpignan, 66860 Perpignan Cedex, FRANCE

E-mail address: horvath@univ-perp.fr

Département de Mathématiques, Université des Antilles et de la Guyane, 97159 Pointe-À-Pitre Cedex, Guadeloupe, France

E-mail address: lassonde@univ-ag.fr 